OPEN ACCESS

\section{Healthy lifestyle and life expectancy free of cancer, cardiovascular disease, and type 2 diabetes: prospective cohort study}

\author{
Yanping Li, ${ }^{1}$ Josje Schoufour, ${ }^{2,3}$ Dong D Wang, ${ }^{1}$ Klodian Dhana, ${ }^{1,4}$ An Pan, ${ }^{5}$ Xiaoran Liu, ${ }^{1}$ \\ Mingyang Song, ${ }^{1,6,7,8}$ Gang Liu, ${ }^{1,9}$ Hyun Joon Shin, ${ }^{10}$ Qi Sun, ${ }^{1,11}$ Laila Al-Shaar, ${ }^{1}$ Molin Wang, ${ }^{6}$ \\ Eric B Rimm, ${ }^{1,11}$ Ellen Hertzmark, ${ }^{12}$ Meir J Stampfer, ${ }^{1,6,11}$ Walter C Willett, ${ }^{1,6,11}$ \\ Oscar H Franco, ${ }^{2,13}$ Frank B Hu $u^{1,6,11}$
}

For numbered affiliations see end of the article.

Correspondence to: FB Hu fhu@hsph.harvard.edu (ORCID 0000-0002-0412-2748)

Additional material is published online only. To view please visit the journal online.

Cite this as: $B M J$ 2020;368:16669 http://dx.doi.org/10.1136/bmj.l6669

Accepted: 14 October 2019

\section{ABSTRACT}

OBJECTIVE

To examine how a healthy lifestyle is related to life expectancy that is free from major chronic diseases.

DESIGN

Prospective cohort study.

SETTING AND PARTICIPANTS

The Nurses' Health Study (1980-2014; n=73196) and the Health Professionals Follow-Up Study (1986-2014; $\mathrm{n}=38366$ ).

\section{MAIN EXPOSURES}

Five low risk lifestyle factors: never smoking, body mass index 18.5-24.9, moderate to vigorous physical activity ( $\geq 30$ minutes/day), moderate alcohol intake (women: 5-15 g/day; men 5-30 g/day), and a higher diet quality score (upper 40\%).

MAIN OUTCOME

Life expectancy free of diabetes, cardiovascular diseases, and cancer.

RESULTS

The life expectancy free of diabetes, cardiovascular diseases, and cancer at age 50 was 23.7 years $(95 \%$ confidence interval 22.6 to 24.7 ) for women who adopted no low risk lifestyle factors, in contrast to 34.4 years (33.1 to 35.5) for women who adopted four or five low risk factors. At age 50 , the life expectancy free of any of these chronic diseases was 23.5 (22.3 to 24.7$)$ years among men who adopted no low risk lifestyle factors and 31.1 (29.5 to 32.5 ) years in men who adopted four or five low risk lifestyle factors. For current male smokers who smoked heavily $(\geq 15$ cigarettes/day) or obese men and women (body mass index $\geq 30$ ), their disease-free life expectancies accounted for the lowest proportion ( $\leq 75 \%)$ of total life expectancy at age 50 .

\section{WHAT IS ALREADY KNOWN ON THIS TOPIC}

Modifiable lifestyle factors including smoking, physical activity, alcohol use, body weight, and diet quality affect both total life expectancy and incidence of chronic diseases

Few studies have comprehensively examined how a combination of multiple lifestyle factors may relate to life expectancy free from the major diseases such as diabetes, cardiovascular disease, and cancer

\section{WHAT THIS STUDY ADDS}

A healthier lifestyle was associated with an increased total life expectancy and life expectancy free of cancer, cardiovascular disease, and type 2 diabetes

\section{CONCLUSION}

Adherence to a healthy lifestyle at mid-life is associated with a longer life expectancy free of major chronic diseases.

\section{Introduction}

The average life expectancy in the world has increased substantially in the past few decades. ${ }^{1}$ The aging of the population has led to a high prevalence of chronic diseases such as diabetes, cardiovascular disease, and cancer. Although people live longer, older individuals often live with disabilities and chronic diseases. ${ }^{2}$ People with chronic diseases including cancer, cardiovascular disease, and diabetes have a shorter life expectancy than do their peers without these chronic conditions. ${ }^{3-5}$ Estimates of the loss in life years due to these chronic conditions range from 7.5 to 20 years, depending on the methods used and the characteristics of the study population. ${ }^{3-5}$

Modifiable lifestyle factors including smoking, physical activity, alcohol intake, body weight, and diet quality affect both total life expectancy and incidence of chronic diseases. ${ }^{6-9}$ Studies have shown that smoking, inactivity, poor diet quality, and heavy alcohol consumption contribute up to $60 \%$ of premature deaths and 7.4-17.9 years' loss in life expectancy. ${ }^{7}$ 10-15 Nevertheless, little research has looked at how a combination of multiple lifestyle factors may relate to life expectancy free from the major diseases of diabetes, cardiovascular disease, and cancer. ${ }^{15-19}$

Because estimates of life expectancy free of chronic diseases take into account both morbidity and mortality, these estimates can be useful metrics for health professionals and the general public, as well as enabling policy makers to better estimate future healthcare costs and to plan for healthcare needs. ${ }^{20-22}$ In a previous analysis, we estimated the effect of healthy lifestyles on the overall life expectancy. ${ }^{23}$ In this study, we examine the effect of healthy lifestyle factors on life expectancy free of cancer, cardiovascular disease, and type 2 diabetes, using data from up to 34 years of follow-up in the Nurses' Health Study (NHS) and 28 years of follow-up in the Health Professions Follow-up Study (HPFS).

\section{Methods}

\section{Study population}

This study was embedded in the NHS and the HPFS. The NHS began in 1976, when 121700 female nurses aged 
30-55 years were included and provided information on medical, lifestyle, and other health related variables. ${ }^{24}$ In 1980, 92468 nurses also completed a validated food frequency questionnaire. The HPFS was established in 1986, when 51529 male US health professionals (dentists, optometrists, osteopaths, podiatrists, pharmacists, and veterinarians) aged 40-75 years completed a mailed questionnaire about their medical history and lifestyle. ${ }^{25}$ In both cohorts, self administered questionnaires have been sent every two years to update the information and identify newly diagnosed cases of various diseases. For this analysis, we used 1980 as the baseline for the NHS and 1986 for the HPFS. We excluded participants already diagnosed as having any of the three outcomes (cancer, cardiovascular disease, and diabetes, $n=15118$ ), those with implausible energy intakes (women: $<500$ or $>3500 \mathrm{kcal} / \mathrm{day}$; men: $<800$ or $>4200 \mathrm{kcal} /$ day), and those with missing values for body mass index, physical activity, alcohol, or smoking at baseline ( $n=17317)$, leaving 111562 participants (73196 women and 38366 men) for analysis. Participants who had missing lifestyle factors at baseline had similar baseline characteristics to those without missing information (supplementary table A).

The NHS and HPFS cohorts were followed across the follow-up periods using similar questionnaires on diet, exercise, smoking status, and other factors (questions on the use of postmenopausal hormone replacement therapies and reproduction related questions were asked in the NHS only). Information on age, ethnicity, use of multivitamins, regular use of aspirin, postmenopausal hormone use (NHS only), and the presence or absence of a family history of diabetes, cancer, or myocardial infarction (in first degree relatives) was collected via biennial questionnaires.

\section{Assessment of lifestyle behaviors}

We derived a healthy lifestyle score based on information on five lifestyle factors-diet, smoking, physical activity, alcohol consumption, and body mass index (BMI). Diet was assessed in the NHS and HPFS by using a validated food frequency questionnaire assessing how often, on average, a participant had consumed a specified amount of a list of foods during the previous year. ${ }^{24}$ Quality of diet was assessed using the Alternate Healthy Eating Index (AHEI) score, which is significantly associated with risk of cardiovascular disease and other chronic diseases in the general population. ${ }^{26}$ We defined a healthy diet as an AHEI score in the top $40 \%$ of each cohort distribution. ${ }^{7}$ Physical activity levels were assessed using a validated questionnaire and updated every two to four years. ${ }^{27}$ We estimated the number of hours per week spent in moderate to vigorous activities (including brisk walking) requiring the expenditure of at least 3 metabolic equivalents of task (METs) per hour. We classified low risk as at least 30 minutes of moderate or vigorous activity daily ( $3.5 \mathrm{~h} /$ week). Height and weight were self reported and used to calculate BMI as weight (kg) divided by height $\left(\mathrm{m}^{2}\right)$. We defined a healthy body weight as a BMI in the range of 18.5-24.9.
Self reported smoking status and the number of cigarettes smoked were updated biennially. We defined never smokers as participants who reported never smoking, current smokers as participants who reported active smoking on the questionnaire, and ever smokers as participants who had smoked in the past but did not report active smoking on the time varying questionnaire. On each questionnaire, current smokers were further classified as smoking one to 14,15 to 24 , or 25 or more cigarettes per day. The food frequency questionnaire also collected alcoholic beverage consumption, including red and white wine separately ( 4 ounces, increasing to 5 ounces in 2006), beer (one glass, can, or bottle), and liquor (one drink or shot). We multiplied the amount of alcohol in grams per specified portion size by servings per day, determined the midpoint of the frequency category, and summed across all beverages to estimate the average alcohol consumption (g/day). We defined moderate alcohol consumption as 5-15 g/day for women and 5-30 g/day for men, consistent with the guidelines for moderate alcohol intake in the US. ${ }^{28}$

Our previous studies using objective measurements had documented the validity of these lifestyle data. A previous validation study showed a correlation of 0.97 between self reported weight and weight measured by a technician. ${ }^{25}$ The correlations between physical activity reported in diaries and that in the questionnaire was 0.62 in women and 0.58 in men. ${ }^{27}$

${ }^{29}$ In addition, independent associations have been observed between physical activity and several biomarkers of obesity and cardiovascular disease risk such as high density lipoprotein cholesterol, leptin, and $\mathrm{C}$ peptide. ${ }^{30}$ Validation studies of the food frequency questionnaire with dietary records indicated an average correlation of 0.63 for all nutrients after adjustment for energy intake and variation in the seven day dietary records. ${ }^{24}$ In addition, correlations with nutrient biomarkers in our study population provided objective evidence for the validity of the food frequency questionnaire. ${ }^{31} 32$ Correlation coefficients between the food frequency questionnaire and four single week diet records for ethanol were 0.90 in the NHS and 0.86 in the HPFS. ${ }^{33}$ Ethanol intake assessed through the food frequency questionnaire was also significantly correlated with serum high density lipoprotein cholesterol $(r=0.33$ for NHS; $r=0.38$ for HPFS). Another validation study among 2485 women participating in the NHS reported that smoking level was strongly associated with toenail nicotine concentration $(r=0.63) .^{34}$

For each low risk lifestyle factor, the participant received a score of 1 if he or she met the criterion for low risk, and 0 otherwise. The sum of these five scores together gave a final low risk lifestyle score ranging from 0 to 5, with higher scores indicating a healthier lifestyle. As the number of cases among the group with the highest lifestyle score was small, especially mortality among participants with prevalent diseases, we combined participants with four or five low risk factors into one group. 
Because lifestyle factors may affect mortality risk over an extended period of time, to best represent long term lifestyle we applied time varying lifestyle information during follow-up, in which mortality risks were predicted by the repeated measurements of these variables during the follow-up periods. The average response rate for the lifestyle risk factors during followup was approximately $94 \%$ for the NHS and $90 \%$ for the HPFS. We calculated average levels of lifestyle factors by using the latest two repeated measurements for our primary analysis of diet, physical activity, and alcohol consumption. ${ }^{35}$ For non-respondents to both questionnaires, we used the last available value carried forward. For AHEI score and alcohol consumption, we calculated the average on the basis of four year repeated measurements. Smoking status was estimated on the basis of both smoking history and most recent status updated every two years and classified into five categories: never smoking, past smoking, and current smoking of $1-14,15-24$, and 25 or more cigarettes per day. To minimize reverse causality, we applied the lifelong maximum BMI by age at risk. ${ }^{36}$ For example, we applied the maximum value of BMI at age 18 and BMI in 1980 to predict mortality between 1980 and 1982 and the maximum value of BMI at age 18, BMI in 1980, and BMI in 1982 to predict mortality between 1982 and 1984, and so forth.

\section{Ascertainment of deaths and non-fatal chronic diseases}

In the NHS and HPFS, most of the deaths were identified through family members or the postal system in response to the follow-up questionnaires. We searched the National Death Index to identify deaths among all study participants, with a high sensitivity $(97.7 \%)$ and specificity $(100 \%) .^{37}$

Self reported diagnoses of cancer, myocardial infarction, and stroke were collected on biennial questionnaires, and participants who reported a new diagnosis were asked for permission to acquire their medical records and pathologic reports. Study physicians, blinded to exposure information, reviewed medical records to confirm the diagnosis. We included all cancers as outcomes except non-melanoma skin cancer. Non-fatal cardiovascular disease outcomes comprised non-fatal myocardial infarction and non-fatal stroke. We classified non-fatal myocardial infarctions as confirmed if the criteria of the World Health Organization were met specifically on the basis of symptoms and either electrocardiographic changes or elevated cardiac enzyme concentrations. We classified stroke cases according to the criteria of the National Survey of Stroke, which required evidence of a neurologic deficit with a sudden or rapid onset that persisted for more than 24 hours. Pathologically confirmed cerebrovascular conditions that were caused by an infection, trauma, or malignancy were not counted as outcomes. Cancer, coronary heart disease, and stroke events for which confirmatory information was obtained by interview or letter but without access to medical records were also included as outcomes. The overall proportion of cases confirmed by medical records in the NHS and HPFS were $82.9 \%$ for cancer, $74.4 \%$ for coronary heart disease, and $64.5 \%$ for stroke.

Cases of type 2 diabetes were identified by self report and confirmed by a validated supplementary questionnaire. ${ }^{38}{ }^{39}$ For cases before 1998, we applied the National Diabetes Data Group criteria. We used the American Diabetes Association diagnostic criteria for confirmation from 1998 onward. The validation of self reported type 2 diabetes diagnosis in the NHS has been documented previously. ${ }^{3839}$

\section{Statistical analysis}

We used population based multistate life tables to calculate the differences in life expectancy and years lived with and without major chronic diseases for each lifestyle factor and the total lifestyle factor score. To assess the association between the number of low risk factors and life expectancy free of cancer, cardiovascular disease, and type 2 diabetes, we took into account three states (disease free, presence of disease, and death), and three transitions between states (from non-disease to incident disease, from non-disease to mortality among participants free of major chronic disease, and from disease diagnosis to mortality among those with disease). Subsequently, we built multistate life tables allowing for the occurrence of the transitions. As shown in supplementary figure A, all participants started from state 0 at baseline; both the period between state 0 and state 1 (before disease diagnosis) and the period between state 0 and state 2 (without disease) contributed to the estimated life expectancy free of cancer, cardiovascular disease, or type 2 diabetes; whereas only the patients with cancer, cardiovascular disease, or type 2 diabetes after diagnosis (from state 1 to state 2) contributed to the estimated life expectancy in the presence of the major chronic disease.

We built a total of four multistate life tables-one for a combination of cancer, cardiovascular disease, and type 2 diabetes and three for individual diseases. For each multistate life table, we first derived overall transition rates by a single year of age in the NHS and HPFS, separately, irrespective of the lifestyle factors, for three transitions ${ }^{4}$ : mortality among participants free of the diseases, incident diseases, and mortality among those with the diseases. We considered only the first entry into a state and no subsequent disease event, and no reversal of state was allowed. Secondly, we calculated hazard ratios to assess the relation between the number of low risk factors and the three transitions by using Cox proportional hazards analyses. We used separate time varying Cox proportional hazards models to assess the risk of mortality without disease, risk of incident disease, and risk of mortality among participants with cancer, cardiovascular disease, and type 2 diabetes. Thirdly, we calculated proportions of low risk factors among the sub-study population for each transition. Lastly, we used the hazard ratios combined with the overall transition rates and low 
risk factor proportions in the multistate life table to calculate total life expectancy and life expectancy with and without diseases for each group of low risk factors. The multistate life tables started at age 50 years and closed at age 105 years. Models were adjusted for age, ethnicity, current multivitamin use, current aspirin use, status with regard to a family history of diabetes, myocardial infarction, or cancer, and, for women, menopausal status and hormone use.

Considering potential bias resulting from changes in diet after the diagnosis of certain diseases, we did a sensitivity analysis in which we stopped updating lifestyle factors at the beginning of the interval in which the participant was diagnosed as having cancer, cardiovascular disease, or diabetes. In another sensitivity analysis, we further classified the past smokers according to the years since smoking cessation.

We used SAS version 9.3 to analyze the data. Statistical significance was set at a two tailed $P$ value of less than 0.05. We used Monte Carlo simulation (parametric bootstrapping) with 10000 runs to calculate the confidence intervals of the life expectancy estimation with @RISK 7.5.

\section{Patient and public involvement}

No patients were involved in setting the research question or the outcome measures, nor were they involved in the design and implementation of the study. We plan to disseminate these findings to participants in our annual newsletter and to the general public in a press release.

\section{Results}

Participants with a higher number of low risk lifestyle factors were more likely to use multivitamin supplements and aspirin (table 1). During 2270411 person years of follow-up of women and 930201 person years of follow-up of men, 34383 deaths were recorded (21344 women and 13039 men).

\section{Life expectancies free of cancer, cardiovascular disease, and diabetes in combination}

Total life expectancy at age 50 increased with increasing number of low risk lifestyle factors: from 31.7 years to 41.1 years in women and from 31.3 years to 39.4 years in men (fig 1). Regardless of the number of low risk lifestyle factors, the vast majority of life expectancy after 50 was free of cancer, cardiovascular disease, or diabetes (fig 1). At age 50, life expectancy free of cancer, cardiovascular disease, and diabetes was 23.7 (95\% confidence interval 22.6 to 24.7 ), 26.4 (25.2 to 27.4 ), 29.1 (28.0 to 30.0), 31.8 (30.8 to 32.8), and 34.4 (33.1 to 35.5) years among women who adopted zero, one, two, three, and four or five low risk lifestyle factors, respectively. Life expectancy free of cancer, cardiovascular disease, and diabetes at age 50 was 23.5 (22.3 to 24.7), 24.8 (23.5 to 26.0), 26.7 (25.3 to 27.9), 28.4 (26.9 to 29.7), and 31.1 (29.5 to 32.5) years among men who adopted zero, one, two, three, and four or five low risk lifestyle factors, respectively (table 2). The percentage of life expectancy free of cancer, cardiovascular disease, and diabetes from total life expectancies was $74.8 \%, 77.6 \%, 80.1 \%$, $82.2 \%$, and $83.6 \%$ among women $(75.3 \%, 75.8 \%$, $76.8 \%, 77.9 \%$, and $79.0 \%$ among men) who adopted zero, one, two, three, and four or five low risk lifestyle factors, respectively.

The increasing trend of life expectancy free of cancer, cardiovascular disease, and type 2 diabetes associated with increasing number of low risk lifestyle factors was observed across the lifespan after age 50 (supplementary figure B). In the sensitivity analysis in which we stopped updating lifestyle factors after the diagnosis of cancer, cardiovascular disease, or diabetes, women and men with four or five low risk lifestyle factors gained 10.0 (9.3 to 10.3) and 7.2 (6.4 to 7.9) years in their life expectancy free of the major chronic diseases, respectively, compared with those with zero low risk lifestyle factors.

\section{Life expectancies free of cancer, cardiovascular disease, or diabetes separately}

Women with four or five low risk lifestyle factors had 10.6 (10.0 to 11.3) years' longer life expectancy free of the major chronic diseases than did women with zero low risk lifestyle factors. When we examined the diseases independently, among women, four to five low risk lifestyle factors were associated with 8.3 (7.8 to 8.9) years' longer life expectancy without cancer, 10.0 (9.3 to 10.6) more years without cardiovascular disease, and 12.3 (11.4 to 13.4) more years without diabetes (table 2). Among men, compared with those with zero low risk lifestyle factors, participants with four to five low risk lifestyle factors gained 7.6 (6.8 to 8.4) years' longer life expectancy free of major chronic diseases: 6.0 (5.4 to 6.7) more years without cancer, 8.6 (7.9 to 9.4) more years without cardiovascular disease, and 10.3 (9.6 to 11.1) more years without diabetes (table 2).

\section{Life expectancies in presence of cancer, cardiovascular disease, and diabetes}

Life expectancy in the presence of cancer, cardiovascular disease, and diabetes was not appreciably different across the groups with different numbers of low risk lifestyle factors (fig 1; supplementary figure B), which was due to a smaller number of patients in the low risk group together with a longer survival time after diagnosis of diseases. Compared with the group with zero low risk lifestyle factors, the hazard ratios for those with four or five low risk lifestyle factors and incident cancer, cardiovascular disease, or type 2 diabetes were 0.5 for women and 0.6 for men (supplementary table B), which means a much lower proportion of incident cancer, cardiovascular disease, or type 2 diabetes among participants with four or five low risk lifestyle factors. After diagnosis of these diseases, half of patients with cancer who adopted four or five low risk lifestyle factors survived up to 22.9 years, whereas half of patients with cancer who adopted zero low risk lifestyle factors survived only up to 11.0 years (supplementary figure C, left); we observed a similar differential 


\begin{tabular}{|c|c|c|c|c|c|}
\hline & & No o & ow risk lifestyle & ctorst & \\
\hline & Zero & One & Two & Three & Four or five \\
\hline Nurses' Health Study (1998) & $(n=11749)$ & $(n=25048)$ & $(n=19837)$ & $(n=9079)$ & $(n=2984$ \\
\hline Mean (SD) age, years & $63.3(7.1)$ & $63.6(7.2)$ & $63.7(7.2)$ & $63.8(7.1)$ & $63.6(6.9)$ \\
\hline Mean (SD) body mass index & $31.0(5.3)$ & $29.2(5.5)$ & $26.6(5.0)$ & $24.4(3.5)$ & $23.1(2.1)$ \\
\hline Mean (SD) Alternate Healthy Eating Index score & $43.0(6.8)$ & $46.6(9.2)$ & $51.4(9.8)$ & $55.3(9.3)$ & $58.3(8.1)$ \\
\hline Mean (SD) physical activity, h/week & $0.5(1.0)$ & $0.9(1.8)$ & $1.9(3.0)$ & $3.5(3.8)$ & $5.5(4.3)$ \\
\hline Mean (SD) alcohol consumption, g/day & $5.0(10.8)$ & $4.5(9.1)$ & $5.1(8.1)$ & $6.1(7.4)$ & $7.3(5.5)$ \\
\hline Past smoking & $9086(77.5)$ & $11314(45.2)$ & $6822(34.4)$ & $2658(29.3)$ & $558(18.6)$ \\
\hline Current smoking & $2663(22.5)$ & $3249(13.0)$ & $1654(8.4)$ & $409(4.5)$ & $50(1.7)$ \\
\hline White & $11524(98.1)$ & $24496(97.8)$ & $19289(97.2)$ & $8839(97.4)$ & $2921(97.9)$ \\
\hline Multivitamin use & $5412(46.1)$ & $12437(49.6)$ & $10480(52.8)$ & $5220(57.5)$ & $1765(59.1)$ \\
\hline Regular aspirin use & $5212(44.5)$ & $11457(45.7)$ & $9145(46.1)$ & $4358(47.9)$ & $1535(51.6)$ \\
\hline Family history of diabetes & $3583(30.5)$ & $7394(29.5)$ & $5296(26.7)$ & $2170(23.9)$ & $690(23.1)$ \\
\hline Family history of cancer & $1549(13.3)$ & $3438(13.7)$ & $2721(13.7)$ & $1236(13.6)$ & $419(14.1)$ \\
\hline Family history of myocardial infarction & $3153(26.8)$ & $6273(25.1)$ & $4787(24.1)$ & $2069(22.8)$ & $705(23.6)$ \\
\hline Health Professionals' Follow-up Study (1998) & $(n=4052)$ & $(n=10043)$ & $(n=10706)$ & $(n=7252)$ & $(n=3710)$ \\
\hline Mean (SD) age, years & $64.3(9.1)$ & $64.0(9.2)$ & $63.7(9.1)$ & $63.7(9.1)$ & $63.5(8.9)$ \\
\hline Mean (SD) body mass index & $29.2(3.8)$ & $28.2(3.7)$ & $26.9(3.5)$ & $25.7(3.1)$ & $24.2(2.2)$ \\
\hline Mean (SD) Alternate Healthy Eating Index score & $42.5(7.1)$ & $45.6(9.0)$ & $50.1(10.1)$ & $54.4(10.0)$ & $58.9(8.9)$ \\
\hline Mean (SD) physical activity, h/week & $0.8(1.2)$ & $1.9(3.8)$ & $3.7(5.6)$ & $5.9(6.6)$ & $8.0(7.2)$ \\
\hline Mean (SD) alcohol consumption, g/day & $13.1(21.2)$ & $10.6(15.4)$ & $10.4(12.4)$ & $10.8(10.5)$ & $11.3(8.2)$ \\
\hline Past smoking & $3465(85.4)$ & $6256(62.2)$ & $5258(49.2)$ & $2711(37.4)$ & $624(16.9)$ \\
\hline Current smoking & $587(14.6)$ & $848(8.5)$ & $491(4.6)$ & $172(2.4)$ & $21(0.6)$ \\
\hline White & $3814(94.1)$ & $9441(94.0)$ & $10117(94.5)$ & $6818(94.0)$ & $3526(95.0)$ \\
\hline Multivitamin use & $1520(37.3)$ & $4247(42.3)$ & $5092(47.6)$ & $3804(52.5)$ & $2135(57.6)$ \\
\hline Regular aspirin use & $2020(49.7)$ & $5349(53.3)$ & $6014(56.2)$ & $4250(58.6)$ & $2264(61.0)$ \\
\hline Family history of diabetes & $861(21.2)$ & $2090(20.8)$ & $2159(20.2)$ & $1510(20.8)$ & 709 (19.2) \\
\hline Family history of cancer & $1291(31.7)$ & $3445(34.3)$ & $3824(35.7)$ & $2641(36.4)$ & $1422(38.5)$ \\
\hline Family history of myocardial infarction & $1300(32.0)$ & $3143(31.3)$ & $3412(31.9)$ & $2336(32.2)$ & $1248(33.8)$ \\
\hline
\end{tabular}

survival probability for people with cardiovascular disease (supplementary figure $\mathrm{C}$, middle) and diabetes (supplementary figure $\mathrm{C}$, right). The percentage of life expectancy with presence of cancer, cardiovascular disease, or diabetes out of total life expectancy was $25.2 \%, 22.4 \%, 19.9 \%, 17.8 \%$, and $16.4 \%$ among women $(24.7 \%, 24.2 \%, 23.2 \%, 22.1 \%$, and $21.0 \%$ among men) who adopted zero, one, two, three, and four or five low risk lifestyle factors, respectively.

\section{Life expectancies associated with individual lifestyle factors}

We also estimated the life expectancy related to each lifestyle factor separately. A healthy dietary pattern, high levels of physical activity, no smoking, moderate alcohol consumption, and an optimal BMI were all associated with longer life expectancy free of cancer, cardiovascular disease, and diabetes (fig 2). The lowest proportions of years of life expectancy free of cancer, cardiovascular disease, or diabetes as a percentage of total life expectancies were observed among men who smoked heavily ( $\geq 15$ cigarettes/day) and obese men and women (BMI $\geq 30)$, for whom $75 \%$ or less of life expectancy at age 50 years was lived free of these chronic diseases (fig 2).

In the sensitivity analysis, women who quit smoking for more than 10 years and less than 10 years lost 1.7 and 6.0 years, respectively, of life expectancy free of chronic diseases compared with never smokers; for men, this was 1.9 and 2.6 years among those with more than 10 and less than 10 years smoking cessation, respectively (supplementary figure D). When we included only four lifestyle factors without alcohol, women who adhered to all four low risk lifestyle factors had 9.5 years' (men: 8.8 years) longer life expectancy free of the major chronic diseases than did those with none of these factors (supplementary figure $\mathrm{E}$, left). After further inclusion of moderate alcohol consumption, women who adhered to all five low risk factors had 12.5 years' (men: 9.6 years) longer life expectancy free of the major chronic diseases than did those with none of these factors (supplementary figure E, right).

\section{Discussion}

Using data from two large cohort studies, we observed that adherence to a low risk lifestyle was associated with a longer life expectancy at age 50 free of major chronic diseases of approximately 7.6 years in men and 10 years in women compared with participants with no low risk lifestyle factors.

\section{Comparison with other studies}

Our results are consistent with previous studies that estimated the individual or clustering effect of lifestyle related risk factors on life expectancy with 
and without chronic diseases. ${ }^{15-19}$ Ferrucci et al found that participants aged 65 years who had never smoked and had higher levels of physical activity had life expectancies without disability (self reported need for help or inability to perform basic activities of daily living) of 6.7 years (men) and 7.3 years (women) higher than their peers who had ever smoked and had low levels of physical activity. ${ }^{16}$ The Framingham Heart Study showed that achieving high levels of physical activity, having a normal weight, and never smoking was associated with lower risk of cardiovascular disease, higher total life expectancy, and greater number of years lived free of cardiovascular disease. ${ }^{17}$ A large study in Europe found that people with no behavior related risk factors lived six years longer without chronic diseases (cardiovascular disease, cancer, respiratory disease, and diabetes) than did participants with at least two behavior related risk factors (including smoking, physical inactivity, and obesity). ${ }^{18}$ The CHANCES study found that a healthy lifestyle was associated with a 7.4-15.7 years longer
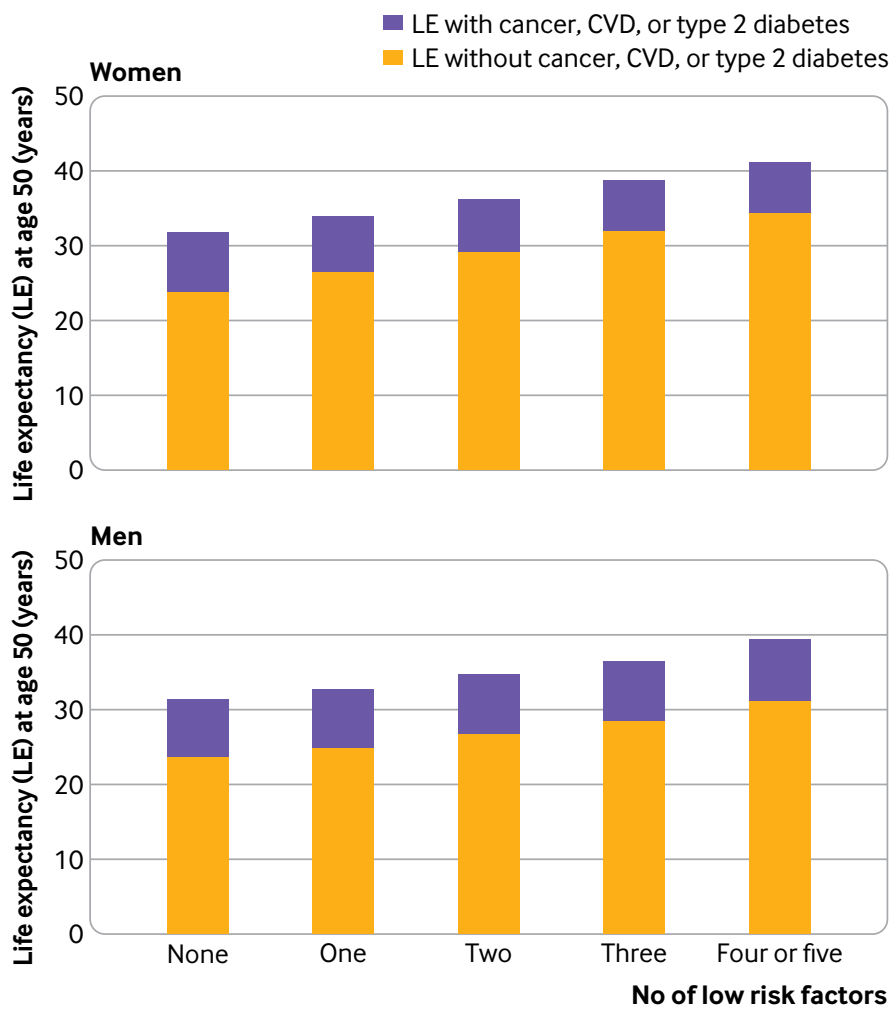

Fig 1 | Estimated life expectancy at age 50 years with and without cancer, cardiovascular disease (CVD), and/or type 2 diabetes among participants of Nurses' Health Study (women) and Health Professionals Follow-up Study (men) according to number of low risk lifestyle factors. Low risk lifestyle factors included cigarette smoking (never smoking), physical activity ( 23.5 hours/week moderate to vigorous intensity activity), high diet quality (upper $40 \%$ of Alternate Healthy Eating Index), moderate alcohol intake of 5-15 g/day (women) or 5-30 g/day (men), and normal weight (body mass index 25 ). Estimates of multivariate adjusted hazard ratios (sex specific) for morbidity and mortality associated with low risk lifestyles compared with people with zero low risk lifestyle factors adjusted for age, ethnicity, current multivitamin use, current aspirin use, family history of diabetes, myocardial infarction, or cancer, and menopausal status and hormone use (women only) life expectancy at age 50 years and that most of these extra life years were free of chronic diseases. ${ }^{15}$ Our study extends previous findings by comprehensively assessing five lifestyle risk factors and three major chronic diseases in combination and by providing broader estimates of longevity and the number of years lived with and without disease in relation to lifestyle factors individually and in combination.

We observed a relatively longer gain in life expectancy free of diabetes associated with a low risk lifestyle than the gained life expectancy free of cancer or cardiovascular disease; this was consistent with the result of different preventable attribution fractions for lifestyle related to specific diseases: in our cohorts, $90 \%$ of diabetes, $80 \%$ of coronary heart disease, $70 \%$ of cardiovascular mortality, and $50 \%$ of cancer mortality were attributable to not following a low risk lifestyle. ${ }^{92338}$ We did not observe a significant difference in life expectancy with cancer, cardiovascular disease, and type 2 diabetes across the number of low risk lifestyle factors. However, this does not necessarily imply that a healthy lifestyle has no effect on patients with these conditions. At the population level, the overall life expectancy living with diseases was determined by both the proportion of the population having the diseases and how long they survived after diagnosis. Compared with those with zero low risk lifestyle factors, men and women with four or five low risk factors had a lower rate of incident cancer, cardiovascular disease, and type 2 diabetes, and they also lived longer after diagnosis of these diseases. This resulted in a paradoxical nondifferential life expectancy in the presence of cancer, cardiovascular disease, and type 2 diabetes across groups with different numbers of healthy lifestyle factors. A healthy lifestyle not only decreased the risk of incident cancer, cardiovascular disease, and type 2 diabetes but also improved the survival after diagnosis of those diseases. ${ }^{41517}$ Thus, the proportion of years of life living with chronic diseases was higher among participants who adhered to an unhealthy lifestyle.

Compared with men, women gained a longer life expectancy free of major chronic diseases through adherence to a low risk lifestyle, resulting from the relatively stronger association between low risk lifestyle and incident cancer, cardiovascular disease, diabetes, and mortality among women than men, as observed in our study. Sex differences in the association between lifestyles and healthy life expectancy were also observed in previous studies, but the reasons for this disparity are not clear. ${ }^{15-19}$ We observed a relatively small difference in life expectancies across different levels of alcohol consumption compared with other individual lifestyle factors. The cardiovascular benefits of moderate alcohol consumption have been consistently observed in large cohort studies, ${ }^{40}$ but alcohol consumption and risk of cancer showed a doseresponse relation. ${ }^{4142}$ Thus, current guidelines do not encourage a non-alcohol drinker to start drinking just for the benefit of preventing cardiovascular disease. 


\begin{tabular}{|c|c|c|c|c|c|}
\hline & \multicolumn{5}{|c|}{ No of low risk lifestyle factors* } \\
\hline & Zero & One & Two & Three & Four or five \\
\hline \multicolumn{6}{|c|}{ Women } \\
\hline \multicolumn{6}{|c|}{ Free of cancer, CVD, or type 2 diabetes: } \\
\hline Life expectancy & $23.7(22.6$ to 24.7$)$ & $26.4(25.2$ to 27.4$)$ & 29.1 (28.0 to 30.0) & 31.8 (30.8 to 32.8$)$ & $34.4(33.1$ to 35.5$)$ \\
\hline Difference & Reference & $2.6(2.3$ to 2.9$)$ & $5.3(5.0$ to 5.6$)$ & $8.1(7.7$ to 8.5$)$ & 10.6 (10.0 to 11.3$)$ \\
\hline \multicolumn{6}{|l|}{ Free of cancer: } \\
\hline Life expectancy & $27.6(26.3$ to 28.7$)$ & 29.7 (28.5 to 30.9$)$ & 31.8 (30.6 to 32.8$)$ & $33.8(32.7$ to 34.8$)$ & 35.9 (34.6 to 37.1$)$ \\
\hline Difference & Reference & $2.2(1.9$ to 2.4$)$ & $4.2(4.0$ to 4.5$)$ & $6.2(5.9$ to 6.6$)$ & $8.3(7.8$ to 8.9$)$ \\
\hline \multicolumn{6}{|l|}{ Free of CVD: } \\
\hline Life expectancy & $30.2(29.9$ to 30.6$)$ & $32.6(32.2$ to 33.0$)$ & $35.0(34.5$ to 35.4$)$ & $37.6(37.0$ to 38.1$)$ & $40.2(39.5$ to 40.9$)$ \\
\hline Difference & Reference & $2.4(2.1$ to 2.7$)$ & $4.7(4.4$ to 5.1$)$ & $7.3(6.9$ to 7.7$)$ & $10.0(9.3$ to 10.6$)$ \\
\hline \multicolumn{6}{|c|}{ Free of type 2 diabetes: } \\
\hline Life expectancy & $28.2(27.0$ to 29.3$)$ & $31.1(30.1$ to 32.0$)$ & 34.3 (33.6 to 35.0$)$ & $37.6(37.0$ to 38.3$)$ & $40.5(39.7$ to 41.3$)$ \\
\hline Difference & Reference & $2.9(2.6$ to 3.2$)$ & $6.1(5.6$ to 6.6$)$ & $9.4(8.7$ to 10.2$)$ & $12.3(11.4$ to 13.4$)$ \\
\hline \multicolumn{6}{|c|}{ Men } \\
\hline \multicolumn{6}{|c|}{ Free of any of cancer, CVD, or type 2 diabetes } \\
\hline Life expectancy & $23.5(22.3$ to 24.7$)$ & $24.8(23.5$ to 26.0$)$ & 26.7 (25.3 to 27.9$)$ & $28.4(26.9$ to 29.7$)$ & $31.1(29.5$ to 32.5$)$ \\
\hline Difference & Reference & $1.2(0.8$ to 1.6$)$ & $3.2(2.7$ to 3.6$)$ & $4.8(4.3$ to 5.4$)$ & $7.6(6.8$ to 8.4$)$ \\
\hline \multicolumn{6}{|l|}{ Free of cancer: } \\
\hline Life expectancy & $27.3(25.8$ to 28.5$)$ & $28.3(26.8$ to 29.6$)$ & $29.7(28.2$ to 31.1$)$ & $31.0(29.4$ to 32.4$)$ & 33.3 (31.6 to 34.8) \\
\hline Difference & Reference & $1.0(0.7$ to 1.3$)$ & $2.5(2.1$ to 2.8$)$ & $3.7(3.3$ to 4.2$)$ & $6.0(5.4$ to 6.7$)$ \\
\hline \multicolumn{6}{|l|}{ Free of CVD: } \\
\hline Life expectancy & $29.0(28.5$ to 29.6$)$ & $30.6(29.9$ to 31.1$)$ & 32.8 (32.1 to 33.4$)$ & 34.5 (33.8 to 35.2) & 37.7 (36.8 to 38.6) \\
\hline Difference & Reference & $1.5(1.1$ to 1.9$)$ & $3.7(3.3$ to 4.2$)$ & $5.5(5.0$ to 6.0$)$ & $8.6(7.9$ to 9.4$)$ \\
\hline \multicolumn{6}{|c|}{ Free of type 2 diabetes: } \\
\hline Life expectancy & $28.0(27.3$ to 28.7$)$ & 29.8 (29.0 to 30.6$)$ & 32.6 (31.7 to 33.4) & 34.9 (34.0 to 35.8) & $38.4(37.3$ to 39.4$)$ \\
\hline Difference & Reference & $1.7(1.3$ to 2.1$)$ & $4.5(4.1$ to 5.0$)$ & $6.9(6.3$ to 7.4$)$ & $10.3(9.6$ to 11.1$)$ \\
\hline \multicolumn{6}{|c|}{$\begin{array}{l}\text { CVD=cardiovascular diseases. } \\
\text { *Included cigarette smoking (never smoking), physical activity ( } \geq 3.5 \mathrm{~h} / \text { week moderate to vigorous intensity activity), high diet quality (upper } 40 \% \text { of Alternate Healthy Eating Index), moderate } \\
\text { alcohol intake of } 5-15 \mathrm{~g} / \text { day (women) or } 5-30 \mathrm{~g} / \text { day (men), and normal weight (body mass index } 18.5-24.9) \text {. Multivariate adjusted hazard ratios (sex specific) for mortality and incident disease } \\
\text { associated with lifestyles factors adjusted for age, ethnicity, current multivitamin use, current aspirin use, family history of diabetes, myocardial infarction, or cancer, and menopausal status and } \\
\text { hormone use (women only). }\end{array}$} \\
\hline
\end{tabular}

\section{Strengths and limitations of study}

Major strengths of this study are the long term follow-up of two large cohorts with low rates of loss to follow-up and the detailed and repeated measurements of lifestyle factors that enabled us to take the dynamic changes in lifestyle factors over time into account. Nevertheless, we also acknowledge several limitations. Firstly, this study was limited by its reliance on self reported lifestyle factors; measurement errors are inevitable. The use of prospectively collected, cumulatively averaged values based on repeated assessments would reduce the effect of random measurement errors. Secondly, residual and unmeasured confounding might also exist even though we controlled for a wide range of potential confounders. However, only a very strong unmeasured risk factor for mortality together with a very large prevalence imbalance among exposure groups could explain such strong findings. ${ }^{43} 44$ Thirdly, we included only cancer, cardiovascular disease, and type 2 diabetes because these are highly prevalent diseases, accounting for most chronic diseases, and their associations with lifestyle factors are well documented. The calculated "disease-free" life expectancy would change if we included other diseases, such as respiratory disease or kidney disease; however, the relative differences in disease-free life expectancy across different lifestyle groups should not materially change. Similarly, we did not take into account the severity and clinical complications of the diseases, which could have affected life expectancies. Fourthly, people may have changed their lifestyle after diagnosis of diseases. Nevertheless, our sensitivity analysis taking into account this potential bias showed similar results. Lastly, because our study populations consist of mostly white health professionals who have adopted relatively healthy behaviors, the results may not be generalizable to other populations. Therefore, further studies are warranted to replicate our findings in other ethnic and racial groups and people with other professional backgrounds. However, the biologic effects of unhealthy behaviors are likely to apply to other populations, and the relative homogeneity of the study populations in educational attainment, health awareness, and socioeconomic status reduces residual confounding and enhances the internal validity.

\section{Conclusion and public health implications}

We observed that a healthier lifestyle was associated with a lower risk of cancer, cardiovascular disease, and diabetes as well as mortality, with an increased total life expectancy and number of years lived free of these diseases. Our findings suggest that promotion of a healthy lifestyle would help to reduce the healthcare burdens through lowering the risk of developing multiple chronic diseases, including cancer, cardiovascular disease, and diabetes, and extending disease-free life expectancy. Public policies for improving food and the physical environment 


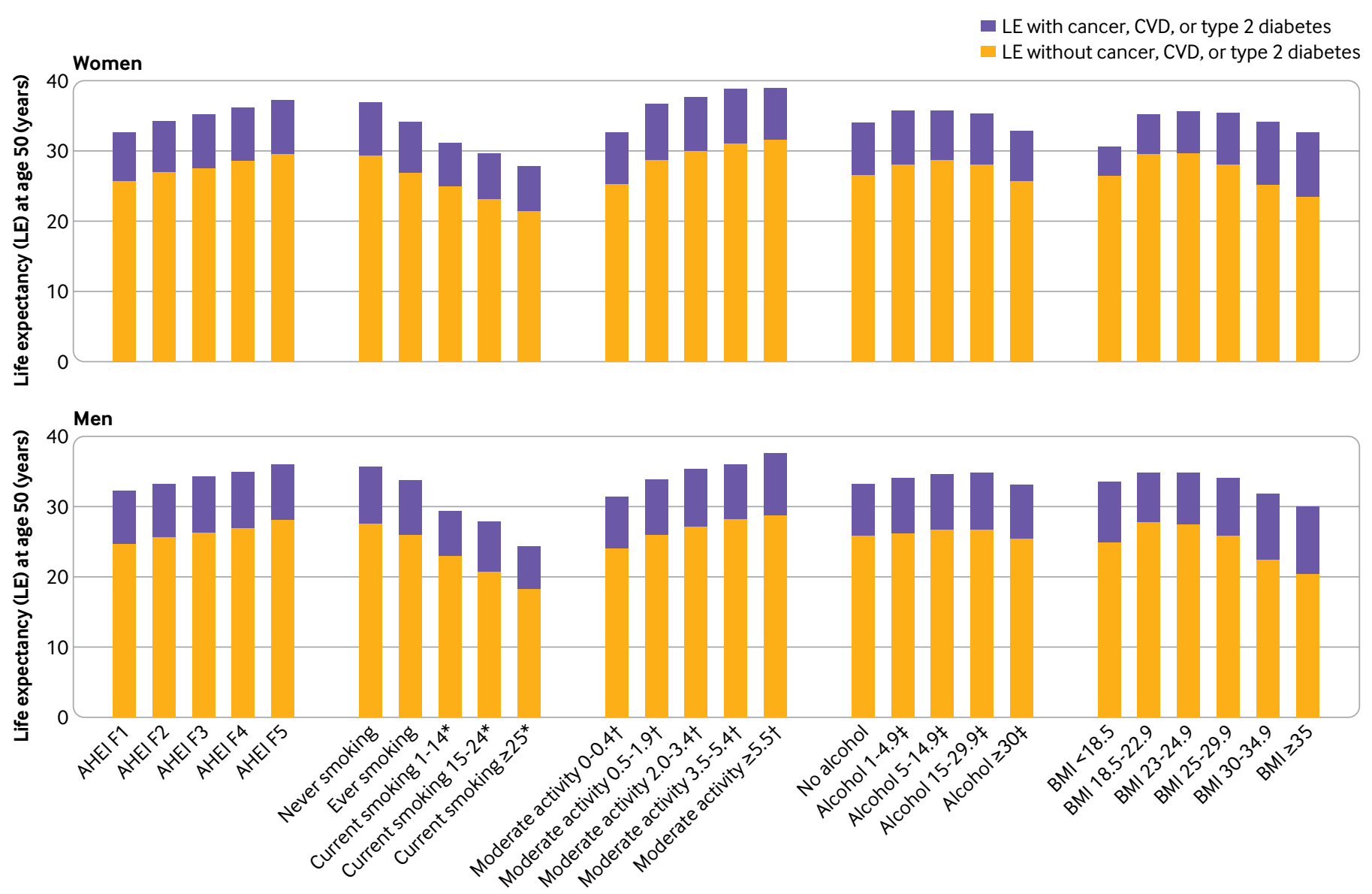

\%LE free of CVD, cancer, and diabetes/total LE

$\begin{array}{lllllllll}\text { Women } & 78.179 .078 .578 .979 .5 & 79.578 .780 .477 .977 .0 & 77.578 .179 .779 .981 .0 & 78.178 .680 .079 .677 .8 & 86.384 .082 .979 .473 .672 .2 \\ \text { Men } & 76.377 .276 .576 .978 .2 & 77.176 .978 .474 .275 .0 & 76.476 .776 .678 .076 .5 & 77.476 .677 .176 .677 .0 & 74.379 .878 .875 .970 .368 .0\end{array}$

Fig 2 | Estimated life expectancy at age 50 years with and without cancer, cardiovascular disease (CVD), and/or type 2 diabetes among participants of Nurses' Health Study (women) and Health Professionals Follow-up Study (men) according to levels of individual lifestyle risk factors. Estimates of multivariate adjusted hazard ratios (sex specific) for morbidity and mortality associated with low risk lifestyles compared with people with zero low risk lifestyle factors adjusted for age, ethnicity, current multivitamin use, current aspirin use, family history of diabetes, myocardial infarction, or cancer, and menopausal status and hormone use (women only). AHEI=Alternate Healthy Eating Index; BMI=body mass index; $\mathrm{F}=$ fifth. *Cigarettes/ day. tHours/week. $¥$ Grams/day

conducive to adopting a healthy diet and lifestyle, as well as relevant policies and regulations (for example, smoking ban in public places or trans-fat restrictions), are critical to improving life expectancy, especially life expectancy free of major chronic diseases.

\footnotetext{
AUTHOR AFFILIATIONS

${ }^{1}$ Department of Nutrition, Harvard T.H. Chan School of Public Health, Boston, MA, USA

${ }^{2}$ Department of Epidemiology, Erasmus Medical Center, Rotterdam, Netherlands

${ }^{3}$ Faculty of Sports and Nutrition, ACHIEVE - Centre of Applied Research, Faculty of Health, Amsterdam University of Applied Sciences, Amsterdam, Netherlands

${ }^{4}$ Department of Internal Medicine, Rush University Medical Center, Chicago, IL, USA

${ }^{5}$ Department of Epidemiology and Biostatistics, Ministry of Education Key Laboratory of Environment and Health, School of Public Health, Tongii Medical College, Huazhong University of Science and Technology, Wuhan, China

${ }^{6}$ Department of Epidemiology, Harvard T.H. Chan School of Public Health, Boston, MA, USA
}

${ }^{7}$ Clinical and Translational Epidemiology Unit, Massachusetts General Hospital and Harvard Medical School, Boston, MA, USA

${ }^{8}$ Division of Gastroenterology, Massachusetts General Hospital, Boston, MA, USA

${ }^{9}$ Department of Nutrition and Food Hygiene, Hubei Key Laboratory of Food Nutrition and Safety, Ministry of Education Key Lab of Environment and Health, School of Public Health, Tongji Medical College, Huazhong University of Science and Technology, Wuhan, China

${ }^{10}$ Division of General Internal Medicine, Division of Global Health Equity, Department of Medicine, Brigham and Women's Hospital, Department of Global Health and Social Medicine, Harvard Medical School, Boston, MA, USA

${ }^{11}$ Channing Division of Network Medicine, Department of Medicine, Brigham and Women's Hospital, Harvard Medical School, Boston, MA, USA

${ }^{12}$ Department of Global Health and Population, Harvard T.H. Chan School of Public Health, Boston, MA, USA

${ }^{13}$ Institute of Social and Preventive Medicine (ISPM), University of Bern, Bern, Switzerland

We thank the participants and staff of the Nurses' Health Study and the Health Professionals Study who contributed data for their valuable contributions, as well as the following state cancer registries for 
their help: AL, AZ, AR, CA, CO, CT, DE, FL, GA, ID, IL, IN, IA, KY, LA, ME, MD, MA, MI, NE, NH, NJ, NY, NC, ND, OH, OK, OR, PA, RI, SC, TN, TX, VA, WA, WY. The authors assume full responsibility for analyses and interpretation of these data.

Contributors: YL and JS contributed equally to the paper. YL, JS, OHF, and FBH had the idea for the study. YL did the data analysis. DDW, KD, MS, ML, EH, MS, WCW, OHF, and FBH provided statistical expertise. YL wrote the first draft of the paper. MS, WCW, and FBH obtained funding. All authors contributed to the interpretation of the results and critical revision of the manuscript for important intellectual content and approved the final version of the manuscript. The corresponding author attests that all listed authors meet authorship criteria and that no others meeting the criteria have been omitted. $\mathrm{YZ}$ and FBH are the guarantors.

Funding: The cohorts were supported by grants of UM1 CA186107, R01 HL034594, R01 HL060712, R01 HL088521, P01 CA87969, UM1 CA167552, and R01 HL35464 from the National Institutes of Health. The funding sources did not participate in the design or conduct of the study; collection, management, analysis or interpretation of the data; or preparation, review, or approval of the manuscript.

Competing interests: All authors have completed the ICMJE uniform disclosure form at www.icmje.org/coi_disclosure.pdf (available on request from the corresponding author) and declare: support from the National Institutes of Health; YL has receiving research support from the California Walnut Commission; AP has received research support from BY-HEALTH outside of the submitted work; FBH has received research support from the California Walnut Commission, and honorariums for lectures from Metagenics and Standard Process, and honorariums from Diet Quality Photo Navigation, outside the submitted work; no other relationships or activities that could appear to have influenced the submitted work.

Ethical approval: The Nurses' Health Study and the Health Professionals Follow-up Study were approved by the institutional review boards at Brigham and Women's Hospital and Harvard T.H. Chan School of Public Health.

Data sharing: No additional data available.

Transparency statement: The lead author (YL) affirms that the manuscript is an honest, accurate, and transparent account of the study being reported; that no important aspects of the study have been omitted; and that any discrepancies from the study as planned (and, if relevant, registered) have been explained.

This is an Open Access article distributed in accordance with the Creative Commons Attribution Non Commercial (CC BY-NC 4.0) license, which permits others to distribute, remix, adapt, build upon this work non-commercially, and license their derivative works on different terms, provided the original work is properly cited and the use is noncommercial. See: http://creativecommons.org/licenses/by-nc/4.0/.

1 GBD 2016 Mortality Collaborators. Global, regional, and national under-5 mortality, adult mortality, age-specific mortality, and life expectancy, 1970-2016: a systematic analysis for the Global Burden of Disease Study 2016. Lancet 2017;390:1084-150. doi:10.1016/ S0140-6736(17)31833-0

2 GBD 2015 DALYs and HALE Collaborators. Global, regional, and national disability-adjusted life-years (DALYs) for 315 diseases and injuries and healthy life expectancy (HALE), 1990-2015: a systematic analysis for the Global Burden of Disease Study 2015 Lancet 2016;388:1603-58. doi:10.1016/S0140-6736(16)31460-X

3 Capocaccia R, Gatta G, Dal Maso L. Life expectancy of colon, breast, and testicular cancer patients: an analysis of US-SEER population-based data. Ann Oncol 2015;26:1263-8. doi:10.1093/annonc/mdv131

4 Franco OH, Steyerberg EW, Hu FB, Mackenbach J, Nusselder W. Associations of diabetes mellitus with total life expectancy and life expectancy with and without cardiovascular disease. Arch Intern Med 2007;167:1145-51. doi:10.1001/archinte.167.11.1145

5 Lee HY, Hwang JS, Jeng JS, Wang JD. Quality-adjusted life expectancy (QALE) and loss of QALE for patients with ischemic stroke and intracerebral hemorrhage: a 13-year follow-up. Stroke 2010;41:73944. doi:10.1161/STROKEAHA.109.573543

6 Loef $\mathrm{M}$, Walach $\mathrm{H}$. The combined effects of healthy lifestyle behaviors on all cause mortality: a systematic review and meta-analysis. Prev Med 2012;55:163-70. doi:10.1016/j.ypmed.2012.06.017

7 LiY, Pan A, Wang DD, et al. Impact of Healthy Lifestyle Factors on Life Expectancies in the US Population. Circulation 2018;138:345-55. doi:10.1161/CIRCULATIONAHA.117.032047

8 Chiuve SE, Rexrode KM, Spiegelman D, Logroscino G, Manson JE, Rimm EB. Primary prevention of stroke by healthy lifestyle. Circulation 2008;118:947-54. doi:10.1161/ CIRCULATIONAHA.108.781062
9 Stampfer MJ, Hu FB, Manson JE, Rimm EB, Willett WC. Primary prevention of coronary heart disease in women through diet and lifestyle. N Engl / Med 2000;343:16-22. doi:10.1056/ NEJM200007063430103

10 Mokdad AH, Forouzanfar MH, Daoud F, et al. Global burden of diseases, injuries, and risk factors for young people's health during 1990-2013: a systematic analysis for the Global Burden of Disease Study 2013. Lancet 2016;387:2383-401. doi:10.1016/S01406736(16)00648-6

11 Tamakoshi A, Tamakoshi K, Lin Y, Yagyu K, Kikuchi S, JACC Study Group. Healthy lifestyle and preventable death: findings from the Japan Collaborative Cohort (JACC) Study. Prev Med 2009;48:486-92. doi:10.1016/i.ypmed.2009.02.017

12 Khaw KT, Wareham N, Bingham S, Welch A, Luben R, Day N. Combined impact of health behaviours and mortality in men and women: the EPIC-Norfolk prospective population study. PLoS Med 2008;5:e12. doi:10.1371/journal.pmed.0050012

13 Manuel DG, Perez R, Sanmartin C, et al. Measuring Burden of Unhealthy Behaviours Using a Multivariable Predictive Approach: Life Expectancy Lost in Canada Attributable to Smoking, Alcohol, Physical Inactivity, and Diet. PLoS Med 2016;13:e1002082. doi:10.1371/ journal.pmed.1002082

14 Li K, Hüsing A, Kaaks R. Lifestyle risk factors and residual life expectancy at age 40: a German cohort study. BMC Med 2014;12:59. doi:10.1186/1741-7015-12-59

15 O'Doherty MG, Cairns K, O'Neill V, et al. Effect of major lifestyle risk factors, independent and jointly, on life expectancy with and without cardiovascular disease: results from the Consortium on Health and Ageing Network of Cohorts in Europe and the United States (CHANCES). Eur J Epidemiol 2016;31:455-68. doi:10.1007/s10654015-0112-8

16 Ferrucci L, Izmirlian G, Leveille S, et al. Smoking, physical activity, and active life expectancy. Am / Epidemiol 1999:149:645-53. doi:10.1093/oxfordjournals.aje.a009865

17 Nusselder WJ, Franco OH, Peeters A, Mackenbach JP. Living healthier for longer: comparative effects of three heart-healthy behaviors on life expectancy with and without cardiovascular disease. BMC Public Health 2009;9:487. doi:10.1186/1471-2458-9-487

18 Stenholm S, Head J, Kivimäki M, et al. Smoking, physical inactivity and obesity as predictors of healthy and disease-free life expectancy between ages 50 and 75: a multicohort study. Int J Epidemiol 2016;45:1260-70. doi:10.1093/ije/dyw126

19 Nyberg ST, Batty GD, Pentti J, et al. Obesity and loss of disease-free years owing to major non-communicable diseases: a multicohort study. Lancet Public Health 2018;3:e490-7. doi:10.1016/S24682667(18)30139-7

20 Wood R, Sutton M, Clark D, McKeon A, Bain M. Measuring inequalities in health: the case for healthy life expectancy. J Epidemiol Community Health 2006;60:1089-92. doi:10.1136/jech.2005.044941

21 Epstein D, Jiménez-Rubio D, Smith PC, Suhrcke M. Social determinants of health: an economic perspective. Health Econ 2009;18:495-502. doi:10.1002/hec.1490

22 Leal J, Gray AM, Clarke PM. Development of life-expectancy tables for people with type 2 diabetes. Eur Heart J 2009;30:834-9. doi:10.1093/eurheartj/ehn567

23 Li Y, Pan A, Wang DD, et al. Impact of Healthy Lifestyle Factors on Life Expectancies in the US Population. Circulation 2018;138:345-55. doi:10.1161/CIRCULATIONAHA.117.032047

24 Willett WC, Sampson L, Stampfer MJ, et al. Reproducibility and validity of a semiquantitative food frequency questionnaire. Am J Epidemiol 1985;122:51-65. doi:10.1093/oxfordjournals.aje. a114086

25 Rimm EB, Stampfer MJ, Colditz GA, Chute CG, Litin LB, Willett WC. Validity of self-reported waist and hip circumferences in men and women. Epidemiology 1990;1:466-73. doi:10.1097/00001648199011000-00009

26 Chiuve SE, Fung TT, Rimm EB, et al. Alternative dietary indices both strongly predict risk of chronic disease. J Nutr 2012;142:1009-18. doi:10.3945/jn.111.157222

27 Wolf AM, Hunter DJ, Colditz GA, et al. Reproducibility and validity of a self-administered physical activity questionnaire. Int J Epidemiol 1994;23:991-9. doi:10.1093/ije/23.5.991

28 U.S. Department of Health and Human Services and U.S. Department of Agriculture. 2015 - 2020 Dietary Guidelines for Americans. 8th ed. 2015. https://health.gov/dietaryguidelines/2015/guidelines/.

29 Chasan-Taber S, Rimm EB, Stampfer MJ, et al. Reproducibility and validity of a self-administered physical activity questionnaire for male health professionals. Epidemiology 1996;7:81-6. doi:10.1097/00001648-199601000-00014

30 Fung TT, Hu FB, Yu J, et al. Leisure-time physical activity, television watching, and plasma biomarkers of obesity and cardiovascular disease risk. Am J Epidemiol 2000;152:1171-8. doi:10.1093/ aje/152.12.1171

31 Hunter DJ, Rimm EB, Sacks FM, et al. Comparison of measures of fatty acid intake by subcutaneous fat aspirate, food frequency 
questionnaire, and diet records in a free-living population of US men. Am J Epidemiol 1992;135:418-27. doi:10.1093/oxfordjournals.aje. a116302

32 Garland M, Sacks FM, Colditz GA, et al. The relation between dietary intake and adipose tissue composition of selected fatty acids in US women. Am J Clin Nutr 1998;67:25-30. doi:10.1093/ ajcn/67.1.25

33 Giovannucci E, Colditz G, Stampfer MJ, et al. The assessment of alcohol consumption by a simple self-administered questionnaire. Am J Epidemiol 1991;133:810-7. doi:10.1093/oxfordjournals.aje. a115960

34 Al-Delaimy WK, Willett WC. Measurement of tobacco smoke exposure: comparison of toenail nicotine biomarkers and self-reports. Cancer Epidemiol Biomarkers Prev 2008;17:1255-61. doi:10.1158/10559965.EPI-07-2695

35 Hu FB, Stampfer MJ, Rimm E, et al. Dietary fat and coronary heart disease: a comparison of approaches for adjusting for total energy intake and modeling repeated dietary measurements. Am IEpidemiol 1999;149:531-40. doi:10.1093/oxfordjournals.aje. a009849

36 Yu E, Ley SH, Manson JE, et al. Weight history and all-cause and cause-specific mortality in three prospective cohort studies. Ann Intern Med 2017:166:613-20. doi:10.7326/M16-1390

37 Rich-Edwards JW, Corsano KA, Stampfer MJ. Test of the National Death Index and Equifax Nationwide Death Search. Am J Epidemiol 1994;140:1016-9. doi:10.1093/oxfordjournals.aje. a117191
38 Hu FB, Manson JE, Stampfer MJ, et al. Diet, lifestyle, and the risk of type 2 diabetes mellitus in women. N Engl J Med 2001;345:790-7. doi:10.1056/NEJMoa010492

39 Manson JE, Rimm EB, Stampfer MJ, et al. Physical activity and incidence of non-insulin-dependent diabetes mellitus in women. Lancet 1991:338:774-8. doi:10.1016/0140-6736(91)90664-B

40 Ronksley PE, Brien SE, Turner BJ, Mukamal KJ, Ghali WA Association of alcohol consumption with selected cardiovascular disease outcomes: a systematic review and meta-analysis. BMJ 2011;342:d671. doi:10.1136/bmj.d671

41 Yen H, Dhana A, Okhovat JP, Qureshi A, Keum N, Cho E. Alcohol intake and risk of nonmelanoma skin cancer: a systematic review and dose-response meta-analysis. Br J Dermatol 2017;177:696-707. doi:10.1111/bjd.15647

42 Chen WY, Rosner B, Hankinson SE, Colditz GA, Willett WC. Moderate alcohol consumption during adult life, drinking patterns, and breast cancer risk. JAMA 2011;306:1884-90. doi:10.1001/ jama.2011.1590

43 Schneeweiss S. Sensitivity analysis and external adjustment for unmeasured confounders in epidemiologic database studies of therapeutics. Pharmacoepidemiol Drug Saf 2006;15:291-303. doi:10.1002/pds.1200

44 Lee WC. Bounding the bias of unmeasured factors with confounding and effect-modifying potentials. Stat Med 2011;30:1007-17. doi:10.1002/sim.4151

\section{Supplementary material}

